\title{
Wave Beams of Short Radio Pulses Generated by Gigawatt Microwave Sources
}

\author{
A. V. Palitsin, M. B. Goykhman, A. V. Gromov, N. F. Kovalev \\ Institute of Applied Physics of the Russian Academy of Sciences, Nizhny Novgorod, Russia, pal@appl.sci-nnov.ru
}

A rising interest to the generation of short pulses is observed lately [1-3]. In relativistic backward wave oscillators (BWO) operating in superradiance regime the peak power of output radiation may exceed the power of electron beam $[1,2]$. Such BWOs are effectively synchronized by the leading edge of accelerating voltage pulse so that the multi-channel systems can be built $[1,2]$. Radiation patterns of these systems consist of overlapping wave beams. The structure of each wave beam is usually close to the fundamental Gaussian beam with linear polarization. Dimensions of the output window of each channel exceed wavelength $\lambda$ because of microwave breakdown on smaller apertures. It means that the distance between channels $d$ is greater (typically much greater) than wavelength

$$
d>>\lambda
$$

and far field radiation pattern has many maximums.

In the report we analyze theoretically the peculiarities of such multi-channel systems in case of radiating the phase synchronized short pulses. Superposition of 2D scalar Gaussian beams (see Fig. 1) has been considered:

$$
\begin{aligned}
E=\operatorname{Re} \sum_{n=1}^{N}\left(\sqrt{\frac{-i k a^{2}}{z-i k a}} \exp [\right. & {\left[k \frac{\left(X-X_{n}\right)^{2}}{2\left(z-i k a^{2}\right)}\right] \times } \\
& \times \exp (i 2 \pi f t-i k z)) .
\end{aligned}
$$

In (2): $E$ is normalized (dimensionless) electric field; $k=2 \pi / \lambda=2 \pi f / c$ is a wavenumber; $f$ is a frequency; $c$ is a velocity of light; $x$ and $z$ are transverse and longitudinal coordinates respectively; $X_{n}$ is a coordinate of the Gaussian beam with index $n$; and $a$ defines the transverse dimension of the beam waist.

Patterns of energy flux density $P$ in far field region have been considered for radiation of:

i. short pulses with the central frequency $f_{0}$ and

Gaussian envelope

$$
E_{p}=\operatorname{Re}\left(\exp \left[-\frac{t^{2}}{2 \tau^{2}}\right] \exp \left(i 2 \pi f_{0} t\right)\right),
$$

where $2 \tau$ is a pulse duration (see Fig. 2).

ii. CW signals with the same frequency $f_{0}$.

For the short pulses the radiation pattern $P_{p}(X)$ is defined as a peak value of energy flux density in the pulse transmitted through the point $X$ in the far field cross section $z=L$. For $\mathrm{CW}$ signals the radiation pattern $P_{f}(X)$ is equal to energy flux density in CW signal at the same cross section $(X, z=L)$.

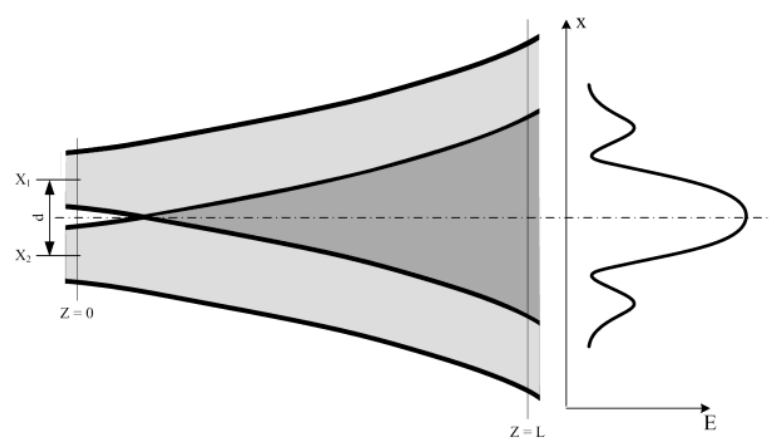

Fig. 1. Superposition of Gaussian beams and distribution of electric field at coordinate $z=L$ in far field region (shown schematically).

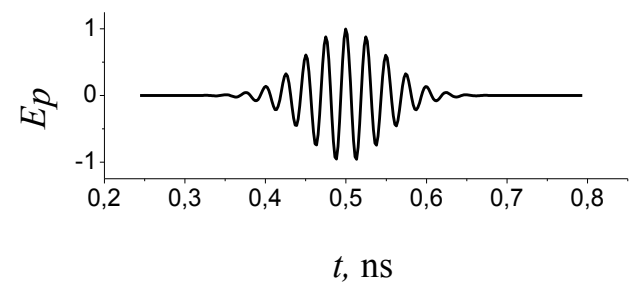

Fig. 2. Short pulse with the central frequency $f_{0}=40 \mathrm{GHz}$ (as an example) and Gaussian envelope.

Summation of two Gaussian beams resulted in small differences of radiation patterns for $\mathrm{CW}$ and short pulses signals as it is shown in Fig. 3. However, all maxima except the main one with highest amplitude are slightly reduced. In case of superposition of 8 Gaussian beams the suppression of side maxima becomes essential (see Fig. 4). Shapes of pulses outside the main maximum are distorted as it is shown in Fig. 5b.

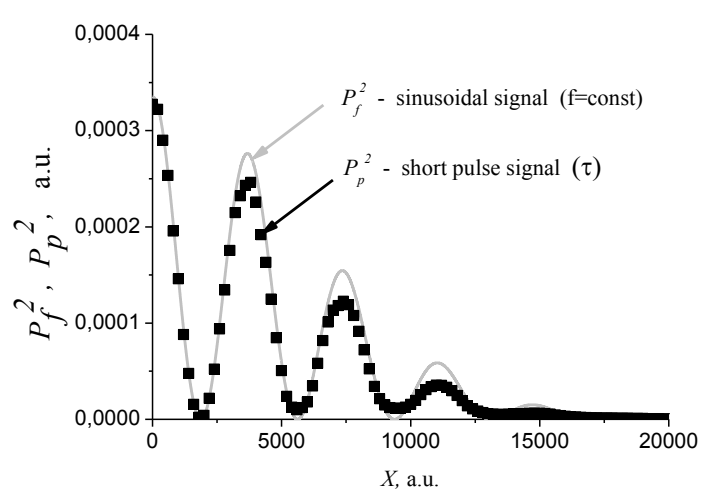

Fig. 3. Radiation patterns of CW (solid gray line) and short pulse (black dots) signals simulated for superposition of two Gaussian beams. 


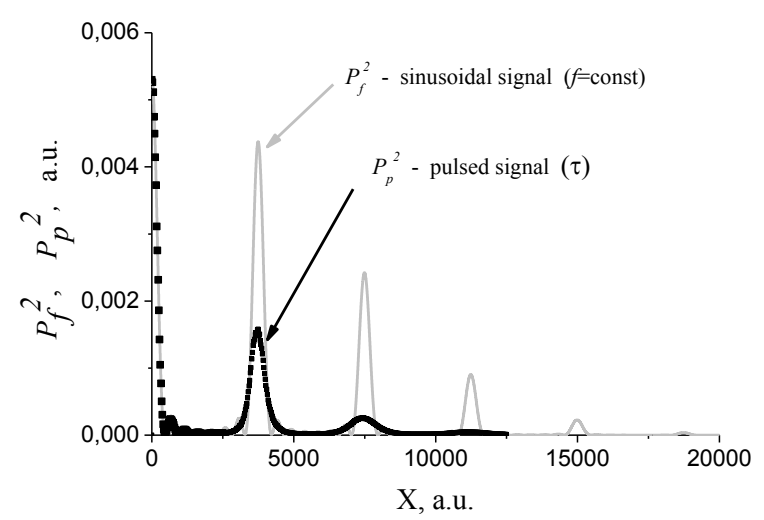

Fig. 4. Radiation patterns of CW (solid gray line) and short pulse (black dots) signals simulated for superposition of 8 Gaussian beams.

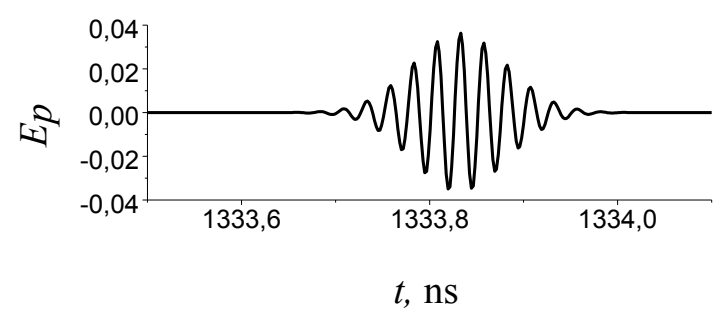

(a)

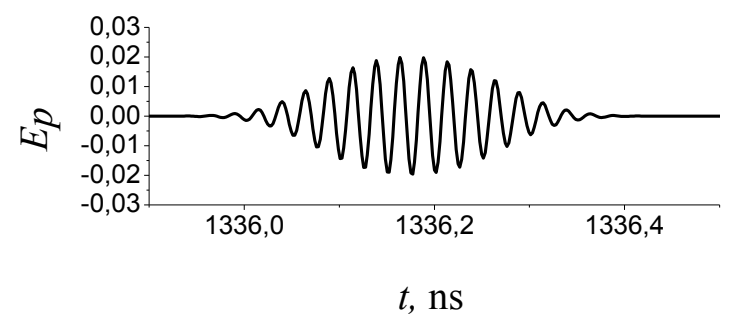

(b)

Fig. 5. Pulses in two points of far field cross section $z=L$. a - in the main maximum $X=0$ and $\mathbf{b}$ - in the first side maximum $X \neq 0$.

The reason of such distortion and accordingly the suppression of side maxima in radiation pattern is a delay of pulses transmitted in different wave beams and observed at the same point $(X \neq 0, z=L)$ in the far field region. It is appropriate to introduce a novel parameter for such multi-beam radiation systems

$$
L_{\text {corr }}=\frac{2 \tau \cdot c}{\sin (\theta)}
$$

a correlation length. Here $2 \tau$ is the pulse duration and $\theta$ is an angle between $z$ axis and the direction to the point of observation (see Fig. 6). For large enough numbers $N$ of wave beams at any point of observation only pulses in wave beams with $X_{n}$ from the range

$$
\Delta X_{n} \leq L_{\text {corr }}
$$

overlap and can be coherently summed. The number $N_{\text {corr }}$ of such beams with overlapping pulses can be named as correlation number.

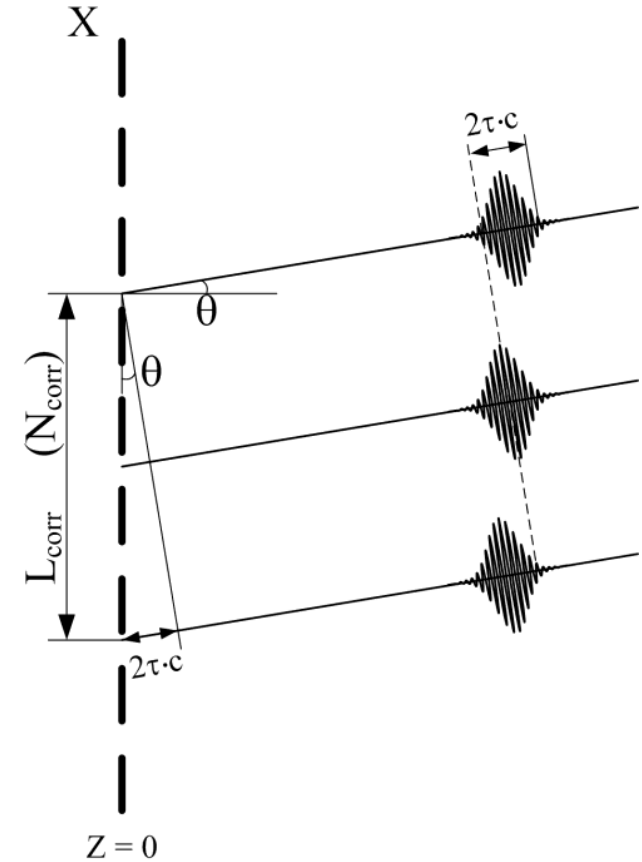

Fig. 6. Explanation for the correlation length $L_{c o r r}$ and correlation number $N_{c o r r}$.

For the angle $\theta=0$ pulses with any durations are overlapping because $L_{\text {corr }}(\theta=0)=\infty$. It is a reason for invariability of main maximum in radiation pattern which is proportional to the square of the total number of wave beams $N^{2}$. For any side maximum $(\theta \neq 0)$ the correlation length is finite (if the pulse duration is finite) and the amplitude of side maxima is proportional to $N_{\text {corr }}{ }^{2}$ as electric fields only from $N_{\text {corr }}$ wave beams are coherently summed. Consequently, for large enough numbers $N$ of the beams the side maxima level of radiation pattern for short pulse signals can be suppressed as low as

$$
\frac{N_{\text {corr }}^{2}}{N^{2}} \rightarrow \frac{1}{N^{2}}
$$

as it is compared to side maxima of $\mathrm{CW}$ radiation pattern.

\section{References}

1. A. Sharypov, A. A. El'chaninov, G. A. Mesyats, M. S.Pedos, I. V. Romancheko et al. Coherent summation of Ka-band microwave beams produced by subgigawatt superradiance backward wave oscillators // Appl. Phys. Lett. 103. 134103 (2013).

2. N.S. Ginzburg, A.W. Cross, A.A. Golovanov, et al. Coherent summation of emission from relativistic cherenkov sources as a way of production of extremely highintensity microwave pulses // IEEE Trans. on Plasma Science. 2016. V. 44. N 4. P. 377-385.

3. N.S.Ginzburg, R.M.Rozental, A.S.Sergeev, A.E.Fedotov, I.V.Zotova, V.P.Tarakanov. Generation of rogue waves in gyrotrons operating in the regime of developed turbulence // PRL 119. 034801 (2017). 